\title{
Growth behavior of plexiform neurofibromas after surgery
}

\author{
Rosa Nguyen, $\mathrm{MD}^{1,2}$, Chadi Ibrahim, MD, BSc${ }^{3}$, Reinhard E. Friedrich, MD, Manfred Westphal, MD5, \\ Martin Schuhmann, $\mathrm{MD}^{6}$ and Victor-Felix Mautner, $\mathrm{MD}^{2}$
}

Purpose: The aim of this study was to analyze growth rate and identify prognostic factors for progression of postoperative plexiform neurofibromas in patients with neurofibromatosis type 1 .

Methods: We measured postoperative tumor volume change per year on magnetic resonance imaging. Linear regression models were applied to identify risk factors for tumor progression.

Results: Fifty-two patients (mean age: 25.4 years (3.2-64.2 years)) with 56 plexiform neurofibromas were analyzed. Initial median tumor volume was $40.3 \mathrm{ml}$ (SD: 1,552 $(0-10,800)$ ). Surgical indications included disfigurement $(n=21)$, pain $(n=20)$, and functional deficits $(n=16)$. Sixteen percent of all cases experienced acute surgical complication, and 13\% showed late complication. Eight patients (19\%; 6 children and 2 adults) with residual tumor had repeat surgery for tumor progression. Median tumor progression was $0.6 \%$ change per year ( $\mathrm{SD} \pm 27.4$; range: -59.2 to 88.1 ) and $2.9 \%$ from baseline (SD \pm

\section{INTRODUCTION}

Plexiform neurofibromas (PNs) are benign peripheral nerve sheath tumors that occur in patients with neurofibromatosis type 1 (NF1). ${ }^{1}$ Approximately $50 \%$ of patients with NF1 have internal PNs evident on whole-body magnetic resonance imaging (MRI). ${ }^{2,3}$ The vast majority of these tumors seem to be congenital in origin and mainly progress during childhood. ${ }^{2,45} \mathrm{PNs}$ arise from Schwann cells of peripheral, visceral, or subcutaneous nerves. ${ }^{6}$ These tumors may manifest as either superficial or deep, and either infiltrating or displacing. Therefore, PNs may cause aesthetic disfigurement and/or functional and neurological deficits depending on location, size, and adjacent anatomical structures that may be involved. ${ }^{7}$ Approximately 50\% of children with internal PNs have tumor-related symptoms in early childhood that correlate with tumor size and location. ${ }^{8}$

To date, management of progressive symptomatic PNs remains medically challenging. PNs appear to be radioinsensitive tumors (unpublished data, own clinical observation), and radiation is avoided given the known risk of radiation-induced secondary malignancies. ${ }^{9}$ Despite emerging molecularly targeted agents, surgery is the only viable treatment option so far.

However, accurate quantification of the progression rate of PNs after surgery is lacking. In previous studies, prognostic
163.9; range $-1,001.3$ to 81.8 ). Patients aged 21 years and younger had the highest progression rate $(P<0.01)$. For every year of age, the mean growth rate decreased by -0.463 mean percent $(P=0.03)$. With age as a continuous variable, age, the site of the tumor, and depth were the only factors associated with tumor progression. Fourteen plexiform neurofibromas ( 10 nodular and 4 diffuse) in 13 patients ( 5 children and 8 adults) were completely resected (by visualization) and did not relapse during observation (mean: 2.9 years; range: $1.1-5.8$ years).

Conclusion: Age, tumor type, location, and depth are helpful to estimate the progression of plexiform neurofibromas after surgery. Patients benefit from elective surgery of small and completely removable plexiform neurofibromas.

Genet Med advance online publication 18 April 2013

Key Words: magnetic resonance imaging; neurofibromatosis type 1; plexiform neurofibroma; surgery; volumetric analysis

indicators of recurrence were identified.$^{10}$ Patients younger than 10 years at the time of surgery; with lesions of the head/neck, face, and trunk; or who received an incomplete resection, had a shorter time to relapse. ${ }^{10,11}$ On the other hand, favorable factors appeared to be gross total resection and low residual tumor volume. ${ }^{10} \mathrm{~A}$ study in seven young patients with small PNs, who underwent complete resection of superficial PNs, demonstrated no relapse after four consecutive years of follow-up. ${ }^{12}$

Surgical complications may be relatively common in NF1 patients given the frequent infiltrating and hypervascular nature of PNs; these include nerve damage, functional impairment, bleeding, and wound-healing abnormalities. ${ }^{13-15}$ Prada et al. ${ }^{14}$ reported sequelae after surgery in approximately $20 \%$ of their studied patients, especially in those with head/neck tumors.

In the current study, we analyze tumor growth rate, prognostic features for tumor progression, and symptoms of postoperative PNs in children and adults with NF1.

\section{MATERIALS AND METHODS}

We retrospectively analyzed clinical and MRI data of 52 children and adults with NF1. NF1 was diagnosed according to the National Institutes of Health Diagnostic Criteria. ${ }^{16}$ After informed consent for participation in the study protocol was obtained, all patients seen for PN at the University Hospital

\footnotetext{
The first two authors contributed equally to this work.

${ }^{1}$ Department of Pediatrics, University of Maryland, Baltimore, Maryland, USA; ${ }^{2}$ Department of Neurology, University Hospital Hamburg-Eppendorf, Hamburg, Germany; ${ }^{3}$ Department of Epidemiology and Public Health, University of Maryland, Baltimore, Maryland, USA; ${ }^{4}$ Department of Maxillofacial Surgery, University Hospital HamburgEppendorf, Hamburg, Germany; ${ }^{5}$ Department of Neurosurgery, University Hospital Hamburg-Eppendorf, Hamburg, Germany; ${ }^{6}$ Department of Neurosurgery, University of Tuebingen, Tuebingen, Germany. Correspondence: Rosa Nguyen (rosi.nguyen@gmail.com)
} 


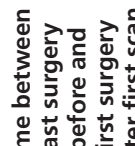

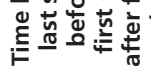

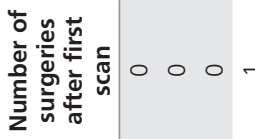

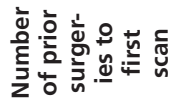

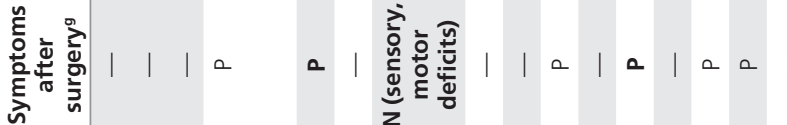

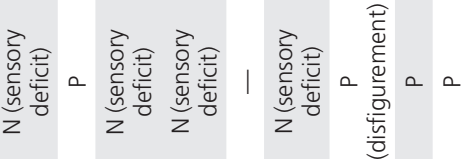

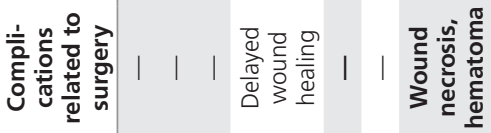

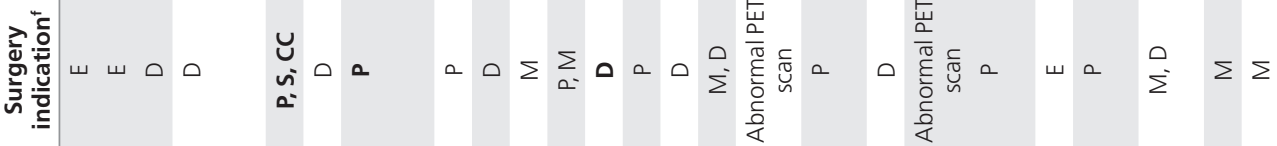

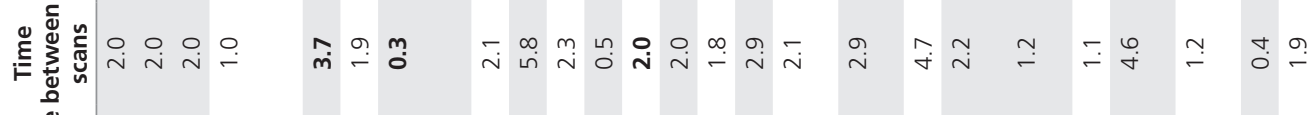

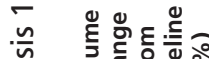

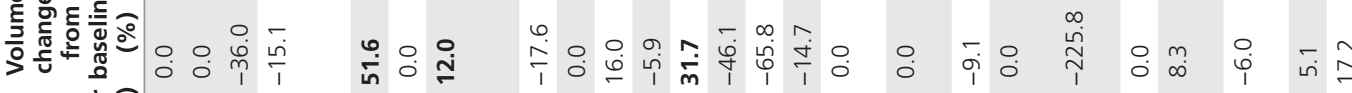

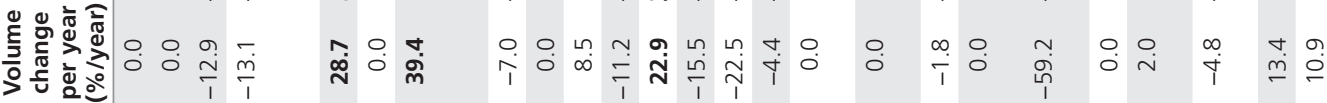

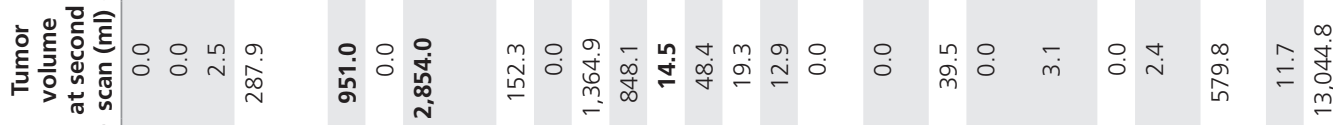

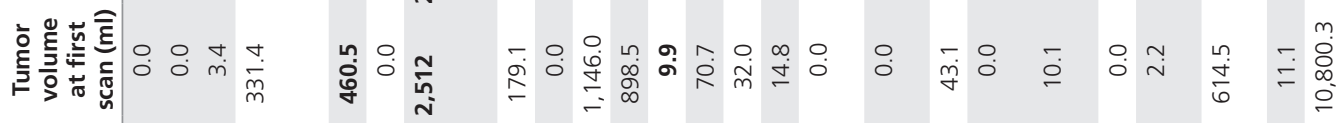

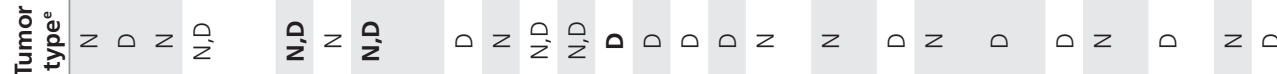

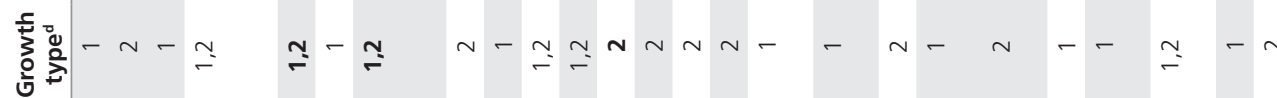

突产

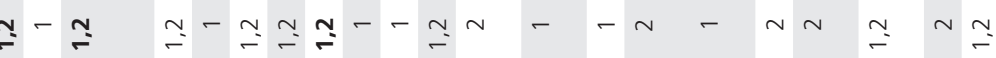

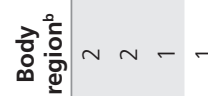

ᄒᄒ

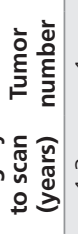

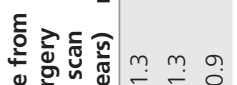

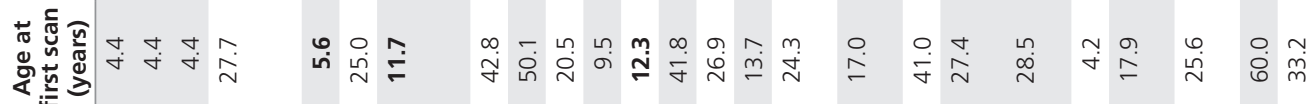

ป 


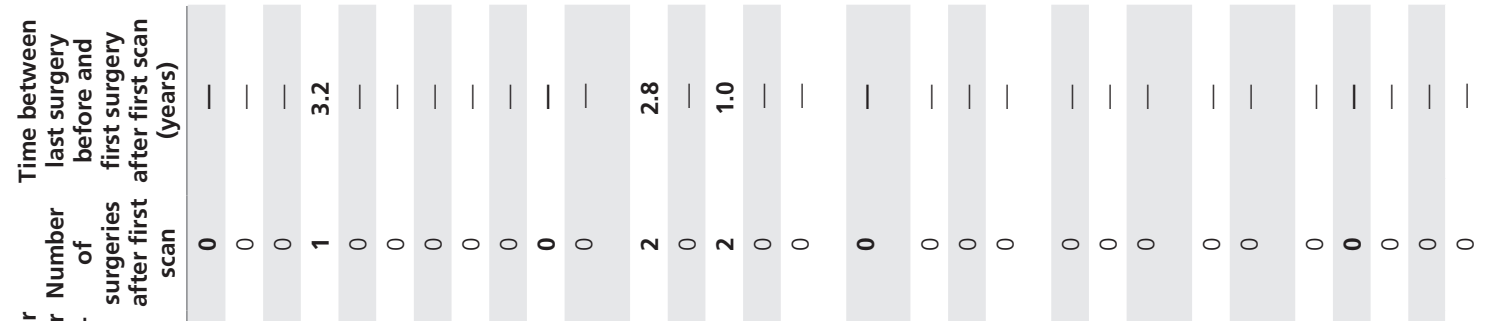

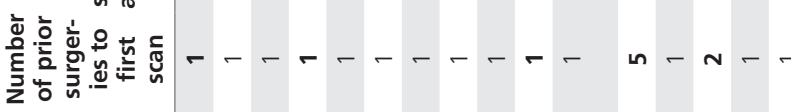

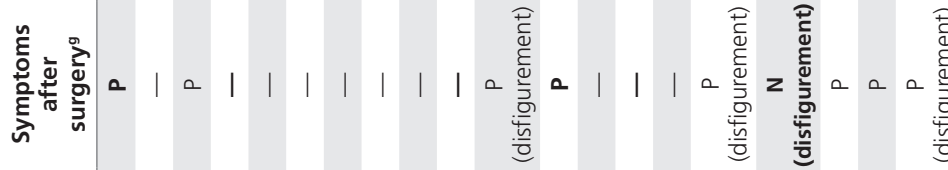

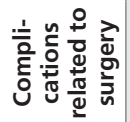

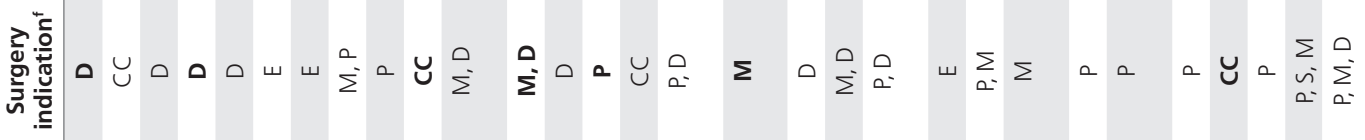

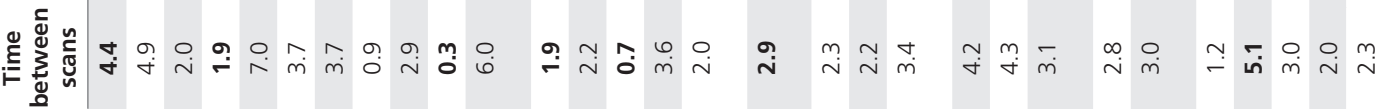

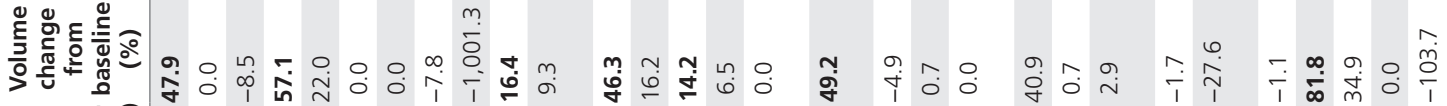

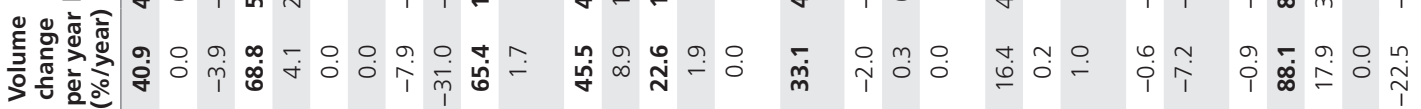

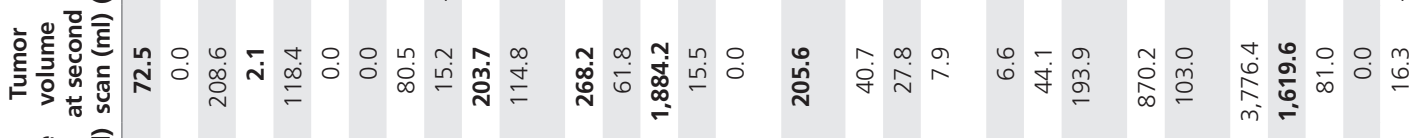

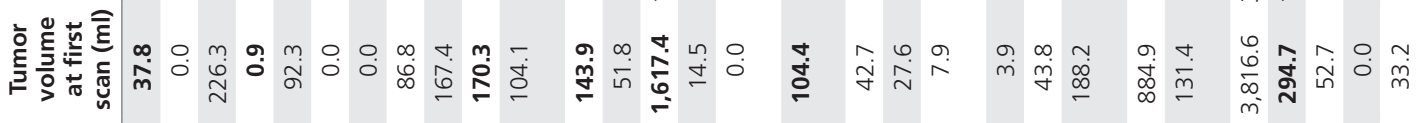

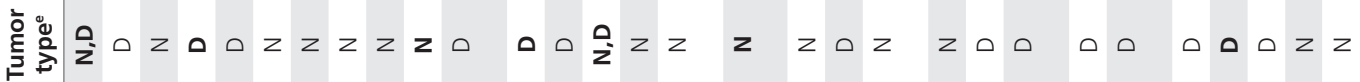

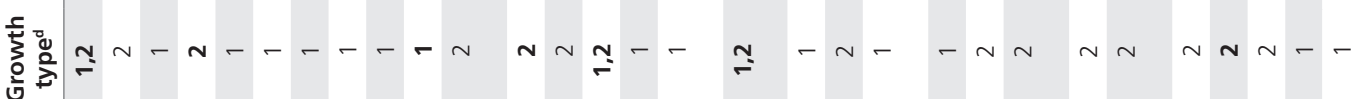

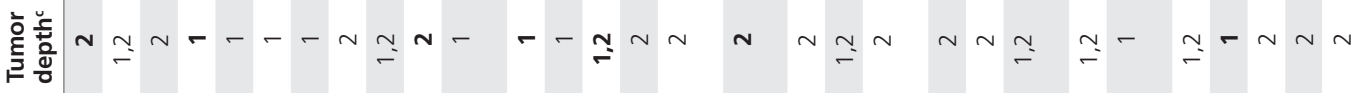

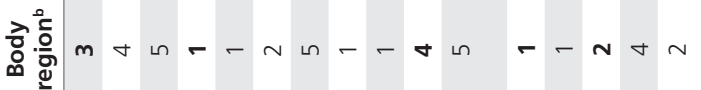

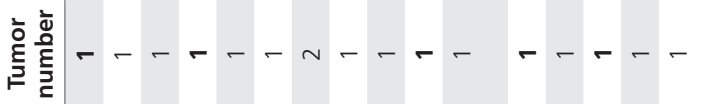

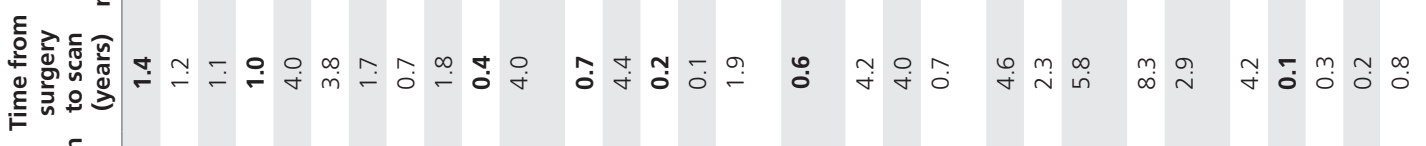



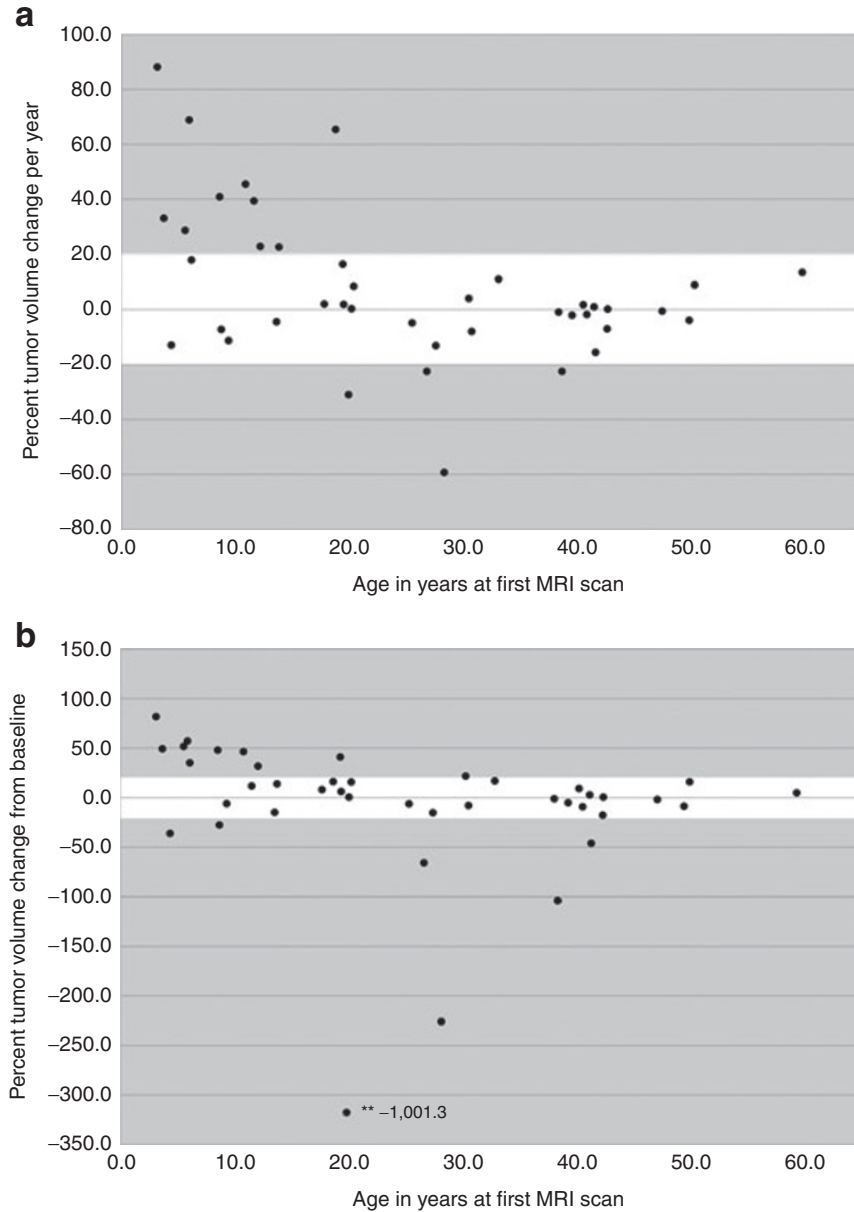

Figure 1 Tumor growth by age. Black dots in the gray areas show more than \pm 20 percentage change per year or from baseline. (a) Black dots represent percentage volume change per year per tumor. (b) Black dots represent percentage volume change from baseline volume per tumor. MRI, magnetic resonance imaging. **Outlier.

Hamburg-Eppendorf between 1999 and 2010 and who had surgical removal of at least one $\mathrm{PN}$ were enrolled. As part of the protocol, all patients had postsurgical MRI studies of their PN. The ethical committee of the Medical Chamber in Hamburg reviewed and approved this project.

\section{Patient and tumor characterization}

All patients were neurologically examined pre- and postoperatively by two of the authors (V.-F.M. and R.E.F.). Preoperatively, patients were examined for tumor-related symptoms that we categorized into (i) functional motor deficit, (ii) functional sensory deficit, (iii) pain, (iv) aesthetic disfigurement, and (v) spinal cord compression. Postoperatively, with the patients, we inquired about and examined for associated residual, resolved, and new tumor-related symptoms and signs, respectively, including the presence of surgical complications. PNs were characterized by their appearance on MRI. In particular, PNs that appeared as a solitary nodule on MRI were labeled as nodular, whereas PNs with a multiform irregular shape were labeled as diffuse. A tumor that contained both features was
Table 2 Regression estimates for the volume change in size in neurofibromas $(n=56)$

\begin{tabular}{|c|c|c|c|c|}
\hline \multirow[b]{2}{*}{ Variable } & \multirow{2}{*}{$\begin{array}{l}\text { Number } \\
\text { of tumors } \\
\text { (total = 56) }\end{array}$} & \multirow{2}{*}{$\begin{array}{c}\text { Number of } \\
\text { patients } \\
(n=52)\end{array}$} & \multicolumn{2}{|c|}{$\begin{array}{l}\text { Percentage volume } \\
\text { change in size }\end{array}$} \\
\hline & & & $\begin{array}{c}\text { Mean } \\
\text { difference }\end{array}$ & $P$ value \\
\hline Age at first scan ${ }^{a}$ & & & -0.463 & 0.0252 \\
\hline Sex & & & & 0.705 \\
\hline Male & 28 & 26 & Ref & \\
\hline Female & 28 & 26 & 2.34 & \\
\hline Site & & & & 0.0347 \\
\hline Head/neck & 17 & 16 & Ref & \\
\hline Chest & 9 & 8 & 6.58 & 0.4897 \\
\hline Abdomen & 9 & 8 & 22.405 & 0.0181 \\
\hline Spine & 9 & 8 & -13.969 & 0.1336 \\
\hline Lower limbs & 9 & 9 & -2.2935 & 0.8126 \\
\hline Upper limbs & 3 & 3 & -0.6554 & 0.9684 \\
\hline \multicolumn{5}{|l|}{ Nature $^{b}$} \\
\hline Nodular & 23 & 20 & -0.454 & 0.9727 \\
\hline Diffuse & 26 & 25 & Ref & \\
\hline Both & 7 & 7 & -15.574 & 0.4434 \\
\hline Deptha $^{a}$ & & & & 0.1488 \\
\hline Superficial & 17 & 16 & 3.247 & 0.6987 \\
\hline Deep & 22 & 19 & Ref & \\
\hline Both & 17 & 17 & 17.907 & 0.0691 \\
\hline Type & & & & 0.5725 \\
\hline Displacing & 25 & 22 & Ref & \\
\hline Invasive & 22 & 21 & -13.709 & 0.3118 \\
\hline Both & 9 & 9 & 19.474 & 0.5472 \\
\hline
\end{tabular}

Ref, reference.

aOn subgroup analysis, age and depth were only significant within those with head and neck tumors (thus this finding is driven by this subgroup).

bFor neurofibromas of the chest: those with both diffuse and nodular nature ("both") had a lower mean volume change percentage $(P=0.0064)$ as compared with those with diffuse nature. Those with nodular only were not significantly different from those with diffuse.

c $P$ value obtained from linear regression $P$ values did not change significantly when age was placed into a model as a categorical variable (21 years and older versus younger than 21 years).

mixed. Growth pattern was determined to be (i) superficial versus deep, (ii) infiltrating versus displacing, (iii) nodular versus diffuse, or (iv) mixed (containing any of the previously mentioned features), as previously described. ${ }^{17}$

\section{Magnetic resonance imaging acquisition}

Serial local short-tau inversion recovery MRI studies were obtained using a 1.5 Tesla Siemens Magnetom 63 SP/Symphony/ Avanto scanner (Siemens Healthcare Diagnostics, Eschborn, Germany). Postoperatively, PNs were scanned in a series of 10-mm slices without skips in between at two different time points. We performed postoperative imaging approximately 1 year after the surgical procedure. From previous clinical experiences, we found that partially resected tumors could falsely 
appear larger if imaging was performed too early postoperatively (apparent size may be due to physiological changes during wound healing). ${ }^{18}$

\section{Volumetric magnetic resonance imaging analysis}

Three-dimensional volumetric tumor analysis was conducted using an MRI-based program for tumor segmentation and volumetric analysis as previously described. ${ }^{19}$ Tumor volume was calculated in milliliters. Because follow-up time periods varied grossly from patient to patient, we also measured postoperative tumor volume percentage change per year in addition to calculating tumor change from baseline as is usually done. Significant tumor progression was defined as changes more than $\pm 20 \%$ per year, as previously described. ${ }^{19}$

\section{Statistical analysis}

Two by two tables were used for frequency calculations. Linear regression models were applied with age as a continuous and categorical variable ( $\leq />21$ years) to identify tumor characteristics associated with tumor growth rate. All calculations were performed with SPSS version 17 (IBM, Chicago, IL). A $P$ value $\leq 0.05$ was considered statistically significant.

\section{Cohort}

\section{RESULTS}

Fifty-two patients (median age: 25.4 years; range: 3.2-64.2 years), 26 females and males each, with 56 PNs were enrolled into the study. Twenty-four patients were younger than 21 years, and 28 individuals were 21 years or older. Initial median tumor volume was $40.3 \mathrm{ml}$ (SD: $\pm 1,552$; range: $0-10,800 \mathrm{ml}$ ). Tumor characteristics are listed in Table 1. The mean time between surgery and the first postoperative MRI scan was 1.7 years (SD: \pm 1.7 ; range: $0.1-8.3$ years). The mean time between first and second postoperative MRI scan was 2.6 years (SD: \pm 1.5 ; range: $0.3-7.0$ years).

\section{Surgical history and complications}

Every patient had undergone at least one surgery prior to the first MRI scan. Fourteen PNs (10 nodular and 4 diffuse) in 13 patients ( 5 children and 8 adults) were completely resected. Surgery of 45 tumors was performed by oral maxillofacial surgeons; nine tumors were resected and/or extirpated by neurosurgeons; and two cases were done in a conjoint procedure. Eight patients had more than two surgeries on their index tumor before their initial MRI scan. Eight patients (19\%; 6 children and 2 adults) with residual tumor had repeated surgery due to tumor progression. Mean time to repeat surgery was 2.6 years with a range of $0.9-5.5$ years. Most frequent surgical indications were aesthetic disfigurement $(n=21)$, pain $(n=20)$, and functional deficits $(n=16)$. Acute surgical complications were bleeding/hematoma $(n=5)$, delayed wound healing $(n=$ 2 ), and necrosis (each $n=1$ ). Surgery yielded complete resolution of symptoms in 24 of 52 patients (46\%), partial relief of symptoms in 5 of 52 patients (10\%), and no change in 16 of 52 patients (31\%). If analyzed by symptoms, pain resolved in 14 of 52 patients (27\%), followed by resolution of functional deficits in 8 of 52 patients (15\%), disfigurement in 6 of 52 patients (12\%), and cord compression in 5 of 52 patients (10\%). In 7 of 56 cases (13\%), patients developed new complaints after surgery, with sensory deficits being the leading symptom (Table 1).

\section{Tumor progression}

We measured tumor growth after surgery (Table 1). Median tumor progression was $0.6 \%$ change per year (SD: \pm 27.4 ; range: -59.2 to 88.1 ) and $2.9 \%$ from baseline (SD: \pm 163.9 ; range $-1,001.3$ to 81.8$)$. Nine of 39 patients (23\%) had tumor progression of $>20 \%$ per year, and only 3 of 39 patients had tumor shrinkage beyond $-20 \%$ per year (Table 1 and Figure 1). In a fully adjusted regression model (Table 2) with age as a categorical variable, patients aged 21 years and younger had the highest progression rate $(P<0.01$; Figure 1$)$. In models stratified according to site, this age effect was maintained only in head/ neck tumors, although cell sizes for several sites were too small to give reliable estimations, particularly for PNs of the upper limbs. With age as a continuous variable, age, the site of the tumor and the depth were the only factors associated with postoperative tumor progression. For every year of age, the mean growth rate decreased by -0.463 mean percent $(P=0.03)$. PNs of the abdomen and PNs that had both superficial and deep areas had higher mean growths as compared with those of the head/neck $(P=0.02)$ and those with only deep areas $(P=0.07)$, respectively. Furthermore, in those with PNs that occurred in the chest, tumor type was significant so that diffuse PNs progressed faster than those with mixed diffuse/nodular features $(P<0.005)$; however, it should be considered that there were only seven tumors of mixed type. Of the 14 PNs (10 nodular and 4 diffuse) in 13 patients ( 5 children and 8 adults) that were completely resected (as determined by visualization), none of the PNs relapsed during observation (mean: 2.9 years; range: 1.1-5.8 years). Preoperative tumor volume in these cases tended to be smaller as compared with the median tumor size of the whole cohort (median: $11.8 \mathrm{ml}$; range: $1.0-246.2 \mathrm{ml}$ ).

\section{DISCUSSION}

This is the first volumetric study that evaluates postoperative growth behavior of PNs in NF1 patients. Although applied methodology in this study allows for more accurate measurement of growth rates, one of the main limitations of the study was that postoperative MRI scans were performed a median of 1.7 years after surgery, and accurate measure of the degree of resection was not feasible.

\section{Postoperative growth behavior of PNs}

From clinical experience, augmented regrowth of PNs after surgery has been a well-known concern among physicians in the past and has led to a cautious approach regarding surgical management of NF1 patients with PNs. In our population, $23 \%$ of the studied tumors regrew significantly after surgery (Table 1, patients in bold), suggesting that one quarter of the studied patients relapsed as determined radiographically. 
Eight individuals (18\%) had prior surgery on their index tumor, indicating that the recurrence rate in our study population varies between 15 and $23 \%$. Of note, all patients with radiographic relapse were younger than 18.9 years and comprise $43 \%$ (9 of 21) of studied patients younger than 21 years. This number is in concordance with findings by Needle et al. $^{10}$, who showed in a pediatric study that of 168 studied PNs, 45\% regrew after surgery.

PNs naturally tend to progress inversely to age. In our cohort, the main progression was found in the subgroup of patients younger than 21 years, resembling the natural history of these tumors. ${ }^{2,45}$ Therefore, postoperative progression of PNs may not be significantly different from the natural growth behavior of these tumors, suggesting that postoperative tumor growth could be unrelated and not promoted by surgery.

\section{Features associated with tumor progression}

In previous studies, young patient age, tumors of the craniofacial area and trunk, and partial resection were found to be associated factors of tumor recurrence. ${ }^{10,13}$ We confirmed these findings and found that depth and diffuse growth type can also be risk factors for fast progression, depending on the primary tumor location. Knowledge about tumor features depending on body sites can be useful for surgical planning and anticipatory guidance of patients regarding surgical outcome.

In previous reports, postsurgical residual tumor volume was reported to be associated with tumor regrowth after surgery. ${ }^{14}$ A retrospective study in 96 children after surgery revealed that near total resection as opposed to debulking was associated with longer intervals between repeated surgeries. ${ }^{13}$ In these studies, tumor progression leading to subsequent symptoms was the most common reason for repeat surgery. Of note, in our cohort, residual tumor volume was not associated with progression rate. However, because progression seems to be inversely correlated with age, it is not surprising that most of the patients with repeat surgery in our cohort were children. Children appear to be a subgroup of patients who are at risk not only for worse surgical outcome but also for increased tumor growth if left untreated. In a recent study, approximately one-third of children with PNs younger than 11.5 years had tumor-related symptoms, which represents an increase of $>50 \%$ of the rate in symptomatic children older than that. Early surgical intervention when the tumor is still small yet completely removable could be beneficial. However, clinical detection of small and asymptomatic PNs remains a challenge because two-third of these tumors are internal. Studies are necessary to evaluate whether early screening with MRI in children with a known diagnosis of NF1 may be warranted and beneficial.

\section{Indications and complications of surgery}

In our patient population, indications for surgery were similar to those of previous reports in the literature. ${ }^{13,14}$ Aesthetic disfigurement was the leading cause of surgery, followed by pain and functional deficits. In approximately $10 \%$ of our patients, predominantly children, elective surgery achieved complete tumor removal and showed no regrowth during observation. Reviewing our cases, complete resection was feasible because the PN was small and superficial; complete resection may be difficult to undertake in patients with huge and infiltrating tumors. Surgery is limited according to the topography of the PN (localization and extension). These factors are of particular importance in the facial region as compared with other parts of the body, e.g., the trunk. For example, the reduction of a bulky lesion is easier to perform at the trunk than at the face, as observed in previous studies. ${ }^{14}$ Hence, we suggest that tumor volume has to be adjusted considering topography. High residual tumor volume is known to be associated with residual symptoms and surgical complications. ${ }^{10}$ Previous reports showed that roughly one-quarter of conducted procedures resulted in new symptoms, with neurological deficits being the leading complaint. ${ }^{13}$ Besides neurological impairment, intraoperative tumor bleeding and wound-healing abnormalities were found to occur in especially large PNs. ${ }^{15}$ Surgical complications observed in our population were similar to those reported. However, the incidence of such complications was higher than previously reported. Approximately one-quarter of our patients had surgery-related complications and newly evolved symptoms after the intervention. Similar to the results of previous studies, neurological deficits were the leading complaint in these patients.

In summary, this is the first study that accurately analyzed the growth rate of postoperative PNs using an MRI-based method. MRI is a valuable tool for postoperative tumor surveillance. The pediatric population and certain tumor features are associated with fast tumor progression and subsequent poorer surgical outcome. Complete resection is most feasible in patients with small and superficial PNs. Further studies are needed to see if early detection of small tumors in childhood and removal of those could improve surgical outcome. Additional investigations are needed to evaluate tumor relapse and growth behavior in the long term.

\section{ACKNOWLEDGMENTS}

The study was supported by the Deutsche Krebshilfe, no. 109713 to V.-F.M. C.I. was supported by NIH award T32 DK067872.

\section{DISCLOSURE}

The authors declare no conflict of interest.

\section{REFERENCES}

1. Korf BR. Plexiform neurofibromas. Am J Med Genet 1999;89:31-37.

2. Nguyen R, Dombi E, Widemann BC, et al. Growth dynamics of plexiform neurofibromas: a retrospective cohort study of 201 patients with neurofibromatosis 1. Orphanet J Rare Dis 2012;7:75.

3. Mautner VF, Asuagbor FA, Dombi E, et al. Assessment of benign tumor burden by whole-body MRI in patients with neurofibromatosis 1. Neuro Oncol 2008;10:593-598.

4. Tucker T, Friedman JM, Friedrich RE, Wenzel R, Fünsterer C, Mautner VF. Longitudinal study of neurofibromatosis 1 associated plexiform neurofibromas. J Med Genet 2009;46:81-85.

5. Dombi E, Solomon J, Gillespie AJ, et al. NF1 plexiform neurofibroma growth rate by volumetric MRI: relationship to age and body weight. Neurology 2007;68:643-647. 
6. Riccardi VM. Neurofibromatosis: phenotype, natural history, and pathogenesis. Johns Hopkins University Press: Baltimore, MD, 1992.

7. Mautner VF, Hartmann M, Kluwe L, Friedrich RE, Fünsterer C. MRI growth patterns of plexiform neurofibromas in patients with neurofibromatosis type 1 . Neuroradiology 2006;48:160-165.

8. Nguyen R, Kluwe L, Fuensterer C, Kentsch M, Friedrich RE, Mautner VF. Plexiform neurofibromas in children with neurofibromatosis type 1 : frequency and associated clinical deficits. J Pediatr 2011;159:652-655.e2.

9. Nakamura JL, Phong C, Pinarbasi E, et al. Dose-dependent effects of focal fractionated irradiation on secondary malignant neoplasms in Nf1 mutant mice. Cancer Res 2011;71:106-115.

10. Needle MN, Cnaan A, Dattilo J, et al. Prognostic signs in the surgical management of plexiform neurofibroma: the Children's Hospital of Philadelphia experience, 1974-1994. J Pediatr 1997;131:678-682.

11. Donner TR, Voorhies RM, Kline DG. Neural sheath tumors of major nerves. J Neurosurg 1994;81:362-373.

12. Friedrich RE, Schmelzle R, Hartmann M, Fünsterer C, Mautner VF. Resection of small plexiform neurofibromas in neurofibromatosis type 1 children. World J Surg Oncol 2005;3:6.
13. Prada CE, Rangwala FA, Martin LJ, et al. Pediatric plexiform neurofibromas: impact on morbidity and mortality in neurofibromatosis type 1. J Pediatr 2012:160:461-467.

14. Wise JB, Cryer JE, Belasco JB, Jacobs I, Elden L. Management of head and neck plexiform neurofibromas in pediatric patients with neurofibromatosis type 1. Arch Otolaryngol Head Neck Surg 2005;131:712-718.

15. Canavese F, Krajbich Jl. Resection of plexiform neurofibromas in children with neurofibromatosis type 1. J Pediatr Orthop 2011;31:303-311.

16. Neurofibromatosis. Conference statement. National Institutes of Health Consensus Development Conference. Arch Neurol 1988;45:575-578.

17. Mautner VF, Hartmann M, Kluwe L, Friedrich RE, Fuensterer C. MRI growth patterns of plexiform neurofibromas in patients with neurofibromatosis type 1. Neuroradiology 2006;48:160-165.

18. Deodhar AK, Rana RE. Surgical physiology of wound healing: a review. J Postgrad Med 1997:43:52-56.

19. Solomon J, Warren K, Dombi E, Patronas N, Widemann B. Automated detection and volume measurement of plexiform neurofibromas in neurofibromatosis 1 using magnetic resonance imaging. Comput Med Imaging Graph 2004;28:257-265. 